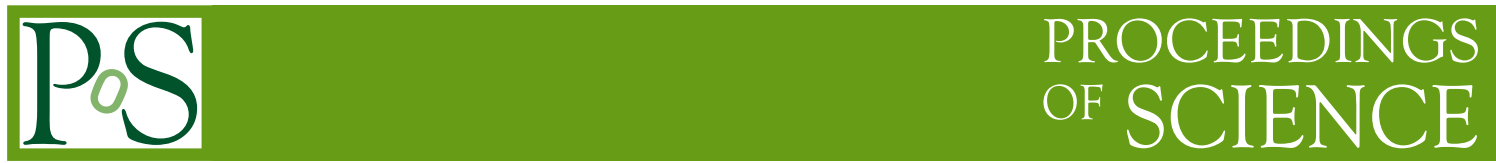

\title{
Production and properties of $2+1$ flavor DWF ensembles
}

\author{
RBC and UKQCD Collaborations: D. J. Antonio, ${ }^{a}$ P. A. Boyle, ${ }^{a}$ M. A. Clark, ${ }^{b}$ C. \\ Dawson, ${ }^{c}$ A. Hart, ${ }^{a}$ C. Jung, ${ }^{d}$ M. Lin, ${ }^{e}$ R. Mawhinney, ${ }^{*}$ B. Pendleton, ${ }^{a}$ R. J. Tweedie ${ }^{a}$ \\ ${ }^{a}$ SUPA, School of Physics, University of Edinburgh, King's Buildings, Edinburgh. EH9 3JZ, UK \\ ${ }^{b}$ Center for Computational Science, Boston University, Boston, MA 02215, USA \\ ${ }^{c}$ RIKEN-BNL Research Center, Brookhaven National Laboratory, Upton, NY 11973, USA \\ ${ }^{d}$ Brookhaven National Laboratory, Upton, NY 11973, USA \\ ${ }^{e}$ Department of Physics, Columbia University, New York, NY 10027, USA
}

\begin{abstract}
The RBC and UKQCD collaborations have generated 2+1 flavor ensembles with domain wall fermions (DWF) using the QCDOC computers. These configurations are produced with the Rational Hybrid Monte Carlo (RHMC), which has been refined and improved during this last year's running to speed up production by about a factor of six. This talk deals with the details of the ensemble production, tuning of the RHMC for DWF, the evolution of topology and other foundational aspects of this ensemble.
\end{abstract}

XXIVth International Symposium on Lattice Field Theory

July 23-28, 2006

Tucson, Arizona, USA

\footnotetext{
${ }^{*}$ Speaker.
} 


\section{Introduction}

The domain wall fermion (DWF) formulation gives a lattice regularization of fermions with controllable chiral symmetry breaking at finite lattice spacing. This control comes from varying the extent of the fifth dimension, denoted by $L_{s}$, that is introduced by DWF. Modified gauge actions produce smoother gauge fields, for a given lattice spacing, and this can reduce the residual chiral symmetry breaking at a fixed $L_{s}$. Such modified gauge actions also inhibit changes in global topological charge, using current Hybrid Monte Carlo algorithms [1].

Failure to sample global topological charge can be viewed as a particular kind of finite volume effect. However, local fluctuations in topological charge do have consequences for local physics and it is important that ensembles do see a representative sample of local topological charge fluctuations. In addition, for simulations with finite quark mass, continuity makes it likely that if toplogical near-zero modes are not evolving appreciably in the ensemble, other small eigenvalues of the Dirac operator could suffer a similar fate. These modes are associated with the chiral symmetry breaking physics which dominates the QCD vacuum and the particle content and a poor sampling of these could lead to poor statistics on the important, volume independent, QCD physics. Efficient sampling of global topological charge is likely an indicator that other important QCD modes are being well sampled.

Studying these issues has led the RBC and UKQCD collaborations to choose the Iwasaki gauge action to achieve a reasonable balance between toplogical evolution and a small residual mass for lattice spacings coarser than $\approx 2 \mathrm{GeV}$. Our production running is being done with $2+1$ flavors of DWF, using the Iwasaki gauge action with $\beta=2.13$, on both $16^{3} \times 32 \times 16$ and $24^{3} \times 64 \times 16$ lattices. We find a lattice spacing of $a^{-1}=1.60(3) \mathrm{GeV}$, yielding a spatial extent of 2 and 3 fermis, respectively. We are using three values for the light quark: $m_{l}=0.01,0.02$ and 0.03 . The dynamical strange quark mass is 0.04 and the residual mass we determine to be $m_{\text {res }}=0.00308(3)$. (The analysis leading to these values is given in [2].) Our current simulations employ optimized versions of the Rational Hybrid Monte Carlo (RHMC) algorithm, which have given us a substantial increase in our evolution rate. In this note, we discuss the RHMC improvements and show how variants of the RHMC effect topological evolutions.

\section{RHMC Implementations}

The RHMC algorithm $[3,4]$ uses rational functions to achieve accurate values for the fractional powers of the Dirac operator that appear in 2+1 flavor simulations of DWF QCD. If we let $\mathscr{D}\left(m_{i}\right)=$ $D_{\mathrm{DWF}}^{\dagger}\left(M_{5}, m_{i}\right) D_{\mathrm{DWF}}\left(M_{5}, m_{i}\right)$, then to simulate the strange quark, with mass $m_{s}$, we need to evaluate $\mathscr{D}\left(m_{s}\right)^{1 / 2}$. In addition, dynamical DWF simulations employ a Pauli-Villars regulator to remove the bulk infinity that occurs if $L_{s} \rightarrow \infty$. This field requires the evaluation of $\mathscr{D}(1.0)^{1 / 2}$.

In addition to the rational function approximations for fractional powers of the determinant, our RHMC implementation also allows the use of either the standard leap-frog integrator or the Omelyan integrator. We can also use a variety of time-scales in the molecular dynamics integration, ala Sexton and Weingarten, allowing us to decompose fermionic and gauge forces and optimize their integration step sizes. Additionally, we can use a single stochastic estimator for the ratio of 
determinants in the RHMC, which we found useful for 2 flavor simulations of DWF with the HMC algorithm [5]. For more information about the RHMC, see [6].

Figure 1 shows 3 variants of the RHMC that we have used. In the figure, each term of the form $\operatorname{det}[\cdots]^{z}$ used a separate stochastic estimator in the evolution. RHMC I is the first, simplest implementation. The fractional power terms utilized a rational approximation and the single term in the denominator, without a fractional power, was done with a conventional HMC. RHMC I was used to start our large volume $\left(24^{3}\right)$ simulations. RHMC II uses the Omelyan integrator and puts the lightest poles in the rational approximation on a coarser time scale than the heavy poles. This speeds the simulations up, because the light poles are costly to invert but give a small contribution to the force. Hence they can be evaluated less often. RHMC III uses the Omelyan integrator and the quotient force term, which reduces the force since the stochastic estimator of the ratio is less noisy than the ratio of stochastic estimators. Notice that we have done a Hasenbush preconditioning of the light quarks by the strange quarks.

For RHMC III on our large volume, the light quark integration is done on the coarsest time scale, the strange quark integration is done on a two times finer time scale and the gauge fields on a still finer time scale. With this decomposition, the light quarks are not a large part of the calculational cost. The light quark forces are expensive to calculate, but do not have to be done so often. The strange quark forces are larger, due to the large number of fermionic modes at high energies, but are cheaper to calculate. A simple measure of this effect is that the total conjugate gradient iterations per trajectory are about 35,000, 32,000 and 30,000 for the ensembles with light quark mass of $0.01,0.02$ and 0.03 , respectively. This shows that the light quark inversions are a small part of the total cost and that going to lighter quark masses, on a fixed volume, is much less costly than it has been previously.

\begin{tabular}{|c|c|}
\hline $\begin{array}{l}\text { RHMC I: } 4 \text { RHMC, } 1 \\
\text { HMC and leapfrog } \\
\text { integrator }\end{array}$ & $\frac{\operatorname{det}\left[\mathcal{D}\left(m_{l}\right)\right]^{1 / 2} \operatorname{det}\left[\mathcal{D}\left(m_{l}\right)\right]^{1 / 2} \operatorname{det}\left[\mathcal{D}\left(m_{s}\right)\right]^{1 / 2}}{\operatorname{det}[\mathcal{D}(1.0)]^{1 / 2} \operatorname{det}[\mathcal{D}(1.0)]}$ \\
\hline $\begin{array}{l}\text { RHMC II: } 4 \text { RHMC, } 1 \\
\text { HMC, multi-timescale } \\
\text { Omelyan integrator with } \\
\text { light poles coarser than } \\
\text { heavy }\end{array}$ & 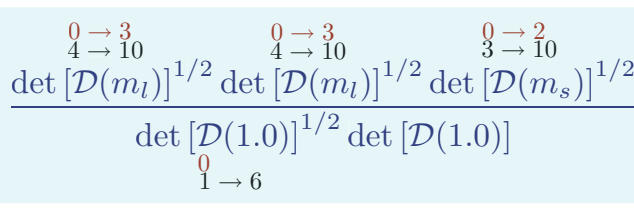 \\
\hline
\end{tabular}

RHMC III: 3 RHMC, 1 HMC, quotient force term, Hasenbush preconditioning and 3 scale Omelyan integrator (HMC, RHMC, gauge)

$$
\operatorname{det}\left[\frac{\mathcal{D}\left(m_{l}\right)}{\mathcal{D}\left(m_{s}\right)}\right] \operatorname{det}\left[\frac{\mathcal{D}\left(m_{s}\right)}{\mathcal{D}(1.0)}\right]^{1 / 2} \operatorname{det}\left[\frac{\mathcal{D}\left(m_{s}\right)}{\mathcal{D}(1.0)}\right]^{1 / 2} \operatorname{det}\left[\frac{\mathcal{D}\left(m_{s}\right)}{\mathcal{D}(1.0)}\right]^{1 / 2}
$$

Figure 1: The three variants of RHMC that we have used most extensively. 


\section{$16^{3} \times 32 \times 16,2+1$ Flavor Topology Evolutions}
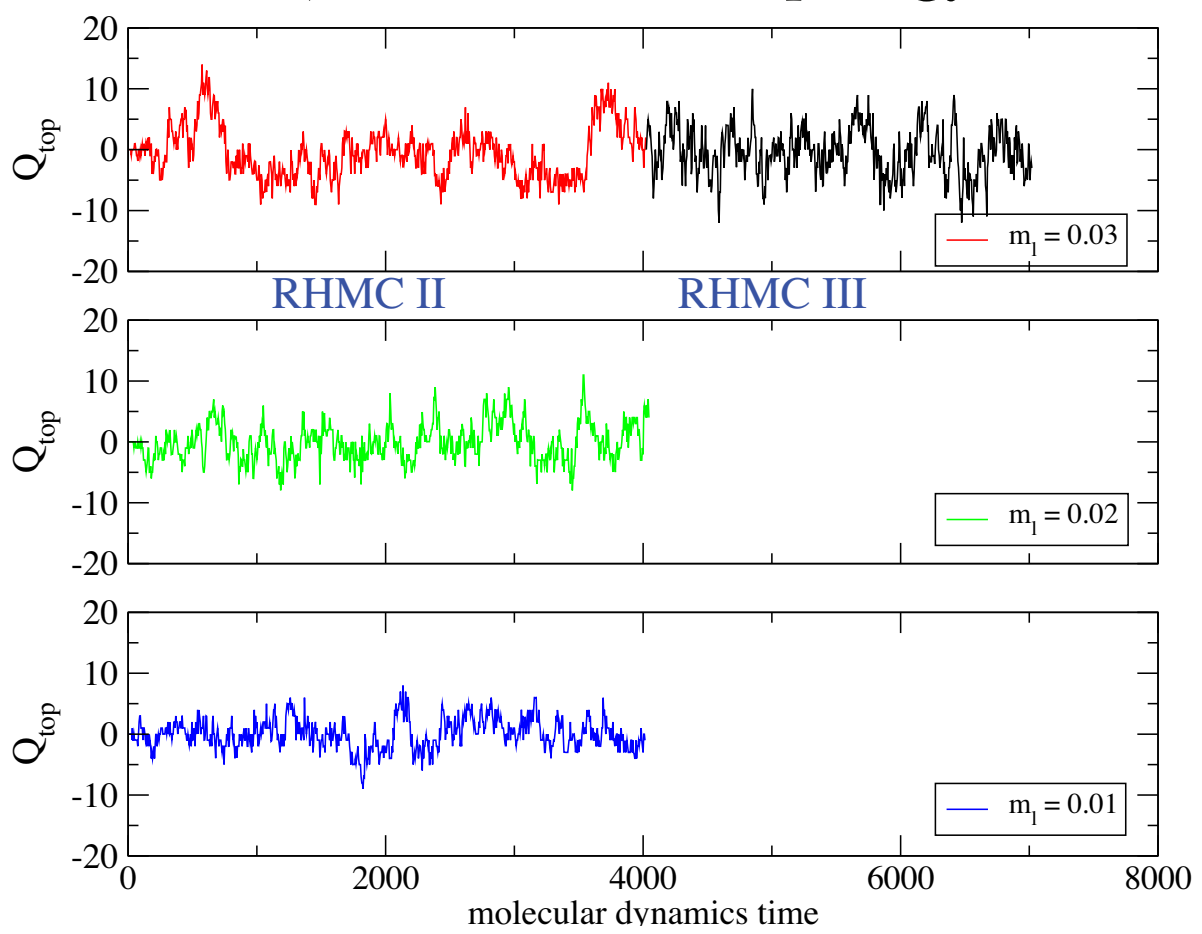

Figure 2: The evolution of global topological charge for our smaller volume.

\section{Small Volume Ensemble}

We have simulated for 4000 units of molecular dynamics time for each light quark mass for our smaller volume. We used the RHMC II algorithm for these simulations and in Figure 2 we plot the evolution of topology. (Here, we measure topological charge using the "5Li" definition of [7].) One notices that the sampling of topology, certainly for the $m_{f}=0.03$ case, seems poor. The width of the topological distribution should decrease with quark mass, which seems generally the case, but the sampling is not very uniform.

For the $m_{f}=0.03$ ensemble, we continued to evolve past 4000 time units, but here we used the RHMC III algorithm. This algorithm uses a single stochastic estimator for the quotient of two determinants, which reduces the noise contribution to the force and allows larger step sizes to be used. From the graph, it is apparent that the topological sampling is much improved as well. The reduction in the noise contribution is clearly helping the algorithm change topology.

In effective mass plots for mesons, using the RHMC II lattices, we see fluctuations that appear outside of the quoted statistical errors. This represents somewhat poor sampling of the phase space for the system and may reflect the same problems seen in the poor topological sampling. With the RHMC III lattices, we are investigating whether the meson effective masses are better behaved. 

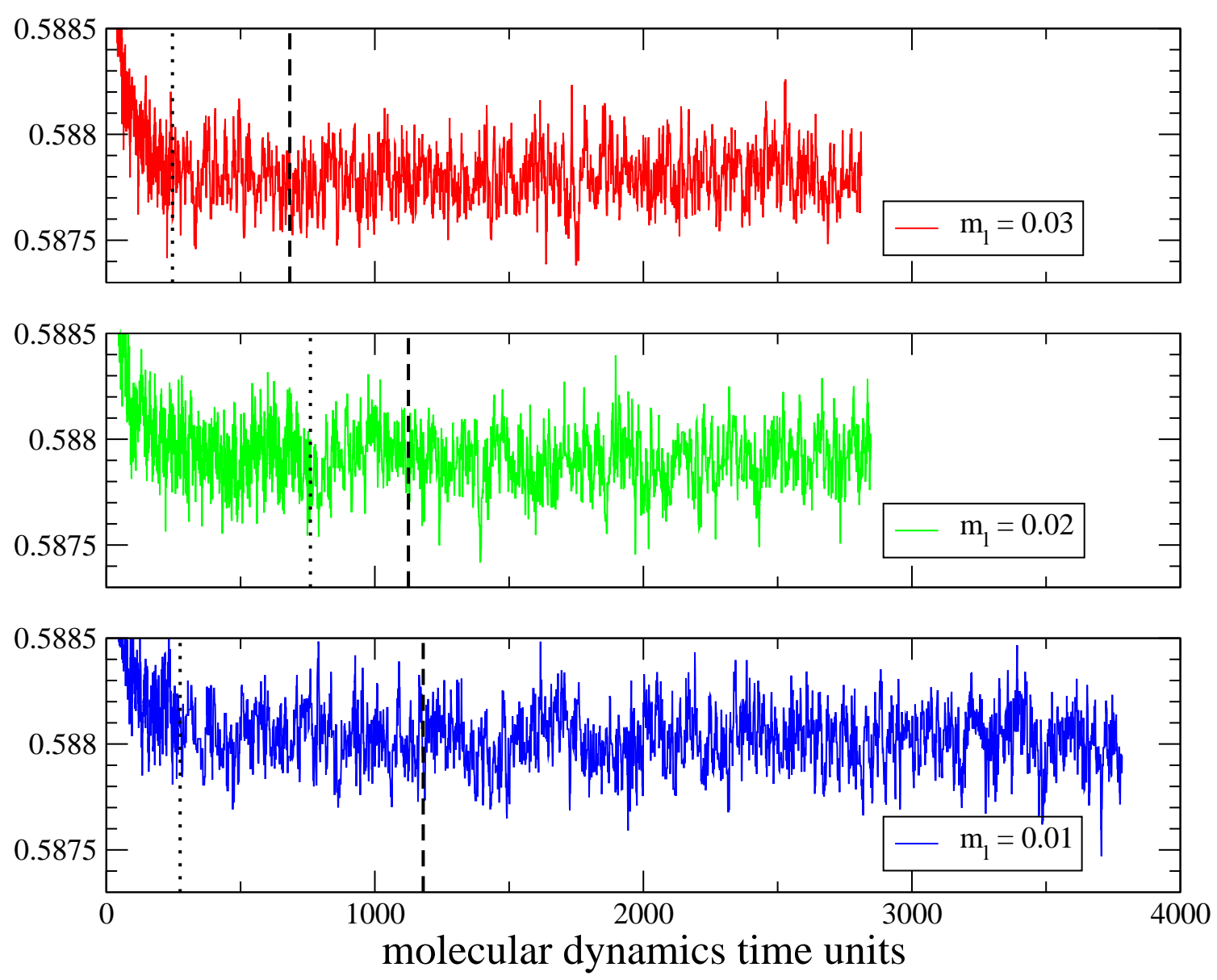

Figure 3: The evolution of the plaquette for our larger, $24^{3} \times 64 \times 16$ volume runs.

\section{Large Volume Ensemble}

While we were running on the smaller volume and working to improve the RHMC, we were also evolving our larger lattices on 4,096 node QCDOC partitions. Initially we used the RHMC I algorithm. The ensemble with light quark 0.01 was taking almost twice as long per trajectory as the ensemble with light quark mass 0.03 . We then switched to the RHMC II algorithm and finally to RHMC III. Figure 4 shows the time history of the plaquette for these ensembles. For times to the left of the dotted line, we used RHMC I; from the dotted to dashed lines we used the RHMC II; to the right of the dashed line we used RHMC III. For the $m_{f}=0.02$ ensemble, it took almost 6 months to generate the lattices to the left of the dotted line and 2 months to generate those on the right of the dashed line.

With the RHMC III algorithm, the difference in the number of conjugate gradient iterations per trajectory between the 0.01 and 0.03 light quark mass cases is only about $10 \%$. For the RHMC I algorithm, with $m_{f}=0.02$, it was taking 8:30 to evolve for 5, 1/2 time unit trajectories. Moving 

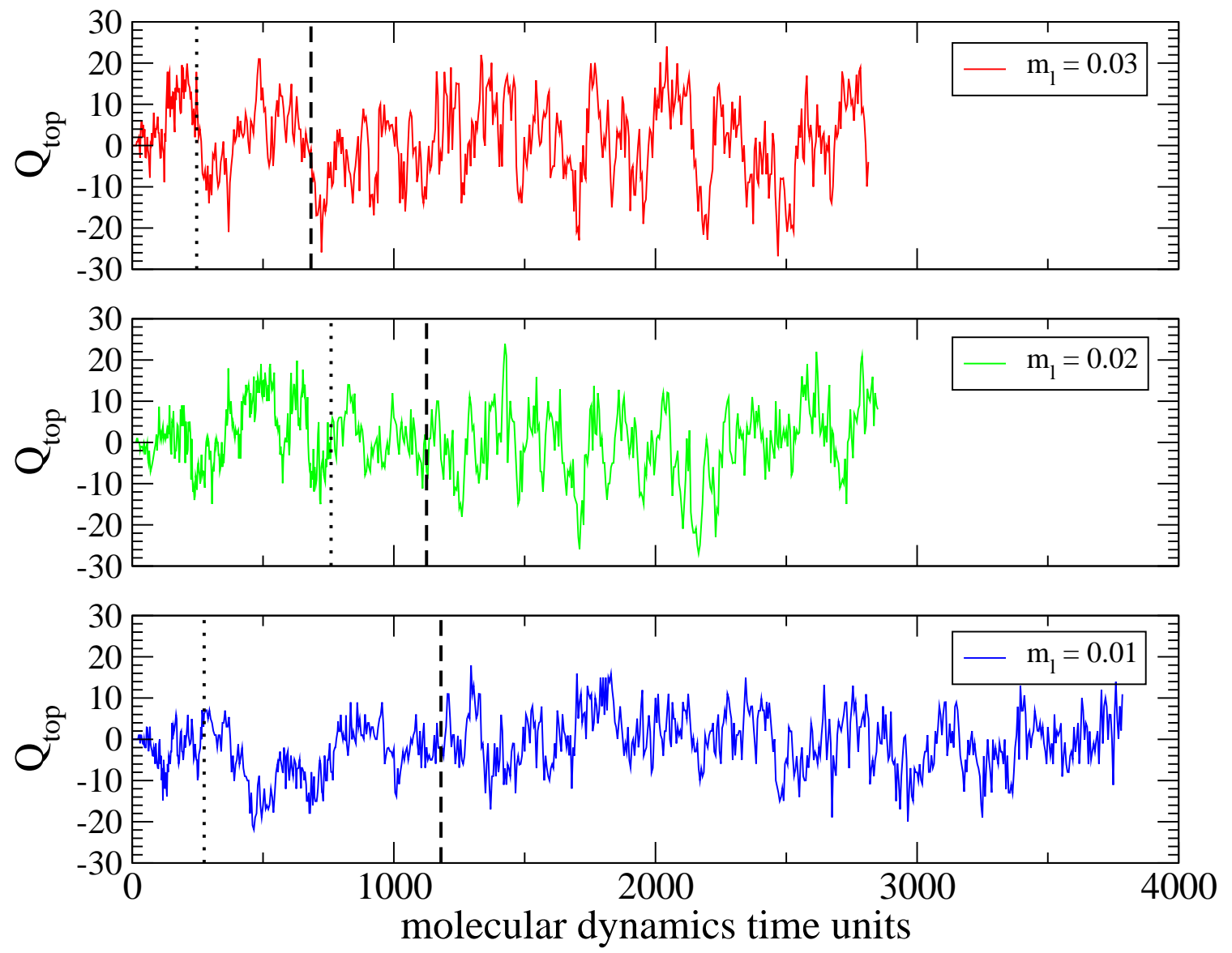

Figure 4: The evolution of the global topological charge for our larger, $24^{3} \times 64 \times 16$ volume runs.

to the RHMC II algorithm, we were able to evolve 5, 1 time unit trajectories in 8:30, where there is an error of \pm 15 minutes on this timing, due to some observable measurements which were running. Finally, with the RHMC III algorithm, we can generate 5, 1 time unit trajectories in 3:10. This is a speed-up of almost 6 for the $m_{f}=0.02$ case. The the $m_{f}=0.01$ case is sped up more, since it was more costly originally.

In Figure 4 we show the evolution of global topology for our large volume lattices. The sampling of topology certainly looks better for the RHMC III case, but the smaller amount of molecular dynamics running with RHMC I and II makes it difficult to be very quantitative. Measurements of many other observables are underway on these lattices and as these complete, we will have a better understanding of whether the evolutions shown are long enough to have well sampled phase space.

\section{Conclusions}

We have been able to markedly speed up our calculations by using the RHMC algorithm, the Omelyan integrator, Hasenbush preconditioning, Sexton-Weingarten mulitple time scale decompositions and the quotient force term. These ideas have produced a viable way to separate the 
expensive-to-calculate-but-small force from the Hasenbush preconditioned light quarks from the cheaper-to-calculate-but-large force from the heavier quarks, which includes the ultraviolet modes in the Dirac operator. It has also been interesting to see that the improvements from these various algorithmic ideas have accumulated to yield a total speedup of a factor of 6 or more. The space of tuning options with this algorithm is quite large and it is likely we have not exhausted all the possibilities.

We have also observed improvements in the sampling of topological sectors as we have improved the speed of the algorithm. As more observables are measured, we will have more information about the quality of these ensembles for other variables.

\section{Acknowledgements}

We thank Peter Boyle, Dong Chen, Mike Clark, Norman Christ, Saul Cohen, Calin Cristian, Zhihua Dong, Alan Gara, Andrew Jackson, Balint Joo, Chulwoo Jung, Richard Kenway, Changhoan Kim, Ludmila Levkova, Xiaodong Liao, Guofeng Liu, Robert Mawhinney, Shigemi Ohta, Konstantin Petrov, Tilo Wettig and Azusa Yamaguchi for developing the QCDOC machine and its software. This development and the resulting computer equipment used in this calculation were funded by the U.S. DOE grant DE-FG02-92ER40699, PPARC JIF grant PPA/J/S/1998/00756 and by RIKEN.

This work was supported by DOE grant DE-FG02-92ER40699, PPARC grant PP/C504386/1 and PPARC grant PP/D000238/1. We thank RIKEN, BNL and the U.S. DOE for providing the facilities essential for the completion of this work.

\section{References}

[1] RBC and UKQCD collaborations, D. Antonio, et. al., Light Hadron Physics in 2+1 Flavour Domain Wall QCD, in preparation.

[2] R. Tweedie, these proceedings, PoS LAT2006 096 (2006).

[3] M. A. Clark and A. D. Kennedy, Nucl. Phys. (Proc. Suppl.) B129 850 (2004), hep-lat/0309084.

[4] M. A. Clark, Ph. de Forcrand and A. D. Kennedy, PoS LAT2005 115 (2006), hep-lat/0510004.

[5] Y. Aoki, et. al., Phys. Rev. D72 114505 (2005), hep-lat/0411006.

[6] M. A. Clark, these proceedings, PoS LAT2006 004 (2006).

[7] Ph. de Forcrand, M. Garcia Perez and I. Stamatescu, Nucl. Phys. (Proc. Suppl.) B47 777 (1996). 